$$
\text { Series A }
$$

I. MATHEMATICA

596

\title{
NONPERSISTENCE OF THE GRENZKREIS PHENOMENON FOR PICK-NEVANLINNA INTERPOLATION ON ANNULI
}

BY

MAURICE HEINS

H E L S I K I 1975

S U O M A L I NEN TIEDEAKATEMIA

doi:10.5186/aasfm.1975.596 
Copyright (C) 1975 by

Academia Scientiarum Fennica

ISSN 0066-1953

ISBN 951-41-0212-6

Communicated 2+ May 1974 


\section{Introduction}

The classical Pick-Nevanlinna interpolation problem has as its object the study of the family of functions analytic on the open unit disk, $\Delta$, taking values of modulus at most one, and having assigned initial Taylor sections of specified orders at assigned points of $\Delta$. Cf. [10], [11], [12], [16]. By the Grenzkreis phenomenon is meant the fact that $W(b)$, the set of values taken by the members of the family at a given point $b(\in \Delta)$ distinct from the assigned points, is a closed circular disk with positive radius in case the family contains more than one member. [Of course, in this case $W(b) \subset \Delta$.$] We recall that W(b)$ is termed the Wertevorrat of the family at $b$.

In this paper we show by counterexamples that the Grenzkreis phenomenon, which is inherent in Pick-Nevanlinna interpolation on $\Delta$, does not persist in the situation of domains of higher topological structure. To obtain the examples we start by considering an annulus and assigning conveniently a Taylor section of order one at one point.

The analysis of the question will make essential use of results obtained by P. R. Garabedian in his study of Pick-Nevanlinna interpolation on a plane region, $\Omega$, of finite connectivity $n(>1)$, no boundary component of which reduces to a point, in the case where there are a finite number $k(\geqq 1)$ of interpolating points and the interpolation conditions are of zero order, i.e. only assign the value of the function [3]. In the cited papar Garabedian showed that if the interpolating family contains more than one member, then exactly one member of the family takes at $b(\in \Omega)$, not an interpolation point, a given value on the frontier of $W(b)$. [ $W(b)$ is understood relative to the situation at hand.] Such a member of the family will be called an extremal in accord with the terminology of Nevanlinna. Cf. [11]. Garabedian further showed that the extremals are maps of finite constant valence of $\Omega$ onto $\Delta$ and that the constant value of the valence of a given extremal is bounded above by $k+(n-1)$. It is remarked that this bound remains valid when the zero order condition is dropped, but the matter is not pursued further.

We shall want to know that the extremals continue to enjoy the property of being uniquely specified by interpolation at $b$ to a given point of $\operatorname{fr} W(b)$ and that they are maps of finite positive constant valence of $\Omega$ onto $\Delta$ when the zero order condition is relaxed. In $\S 8$ we give a general discussion 
of this question for the case of non-compact hyperbolic Riemann surfaces with finite topological characteristics, interpolation being to a finite number of initial sections of specified orders (given in terms of local uniformizers). An extension of Garabedian's upper bound for the valence of the extremal, based on methods due to F. Riesz [15] and the Theorem of Cauchy-Read [14], [7], will be included. [The extension of the Garabedian valence bound for the special "Schwarz Lemma» case (involving one interpolation condition of zero order) was treated by Ahlfors [1], who also observed for the case in question that the contour number gave a lower bound for the valence.] For the purposes of the example which we are examining, Garabedian's upper bound may be shown to persist by a limit argument (cf. § 3). [The limit argument is available generally but would have no special advantage in a general setting.] That a lower bound of the constant value of the valence is $n$ is a direct consequence of the constant valence property of the extremals and the fact that $\Omega$ is $n$-tuply connected. It does not depend on stipulations concerning order of interpolation.

Using a convexity property enjoyed by $W(b)$ and results of [4], which treats Pick-Nevanlinna interpolation on an annulus via uniformization methods, and of [8] we obtain qualitative information concerning $W(b)$ for the case of an annulus when the interpolating family contains more than one member. In fact, then, as we shall see in $\S 6$, either $W^{\prime}(b)$ is a closed circular disk lying in $\Delta$ for all allowed $b$, or is the intersection of two closed circular disks, each a proper part of $\bar{\Delta}$, and such that their circumferences have exactly two points in common, for all allowed $b$. Examples of the first possibility are given in a straightforward way (e.g. the "Schwarz Lemma» situation). That the second possibility is realized follows from the counterexamples.

The convexity property of $W(b)$ in question (which holds in the completely general setting of Pick-Nevanlinna interpolation on a Riemann surface) is that $W(b)$ has the Study property, namely: whenever $\alpha$ is a univalent analytic map of $\Delta$ onto a convex region, $\alpha[W(b)]$ is convex. This follows on considering

$$
\operatorname{inv} \alpha \circ\left[(1-t) \alpha \circ f_{1}+t \alpha \circ f_{2}\right],
$$

where $0 \leqq t \leqq 1$ and $f_{1}$ and $f_{2}$ are interpolating functions. [It is to be observed that in his cited paper Garabedian discerned, using (1.1) with $\alpha$ specialized to conformal automorphisms of $\Delta$, a convexity property of $W(b)$ relative to a class of circular arcs. This latter property is subsumed under the Study property (and may be used to show that $W(b)$ has the Study property).]

The study of $W(b)$ for the general situation of a Riemann surface is doubtless complicated. 


\section{The counterexamples}

We introduce a fixed $r$, satisfying $0<r<1$, and $c$, small and positive. We let $B(c)$ denote the set of functions $f$, analytic on $\mathfrak{A}=$ $\left\{r<|z|<r^{-1}\right\}$, taking values of modulus less than one, and satisfying the interpolation conditions

$$
f(-1)=0, f^{\prime}(-1)=c .
$$

We are concerned with the Wertevorrat $W(1)$ for the present problem, which we shall denote by $W_{c}$ to indicate dependence on the parameter $c$. It is our object to show:

For $c$ sufficiently small $W_{c}$ is a set with non empty interior but is not a circular disk.

It follows that the Grenzkreis phenomenon does not hold for $B(c)$, $c$ small. We shall see, $\S 5$, that the Grenzkreis phenomenon also fails for the class $C(c, h)$ of functions satisfying the conditions imposed on the members of $B(c)$ save that (2.1) is replaced by the two zero order conditions

$$
f(-1)=0, f(-1+h)=c h,
$$

where $c$ is a fixed positive number, suitably small, and $h$ is sufficiently small and positive.

\section{Valence analysis of extremals}

When $c$ is small, $B(c)$ is infinite and $W_{c}$ has a non-empty interior, as we see from a purely elementary consideration of

$$
c(z+1)+d(z+1)^{2} .
$$

We continue supposing that $c$ is so restricted. Given $w \in \operatorname{fr} W_{c}$ we denote by $f_{c, w}$ the extremal of $B(c)$ taking the value $w$ at 1 . We let $v(c, w)$ denote the constant valence of $f_{c, w}$ on $\mathcal{A}$. Since $\mathfrak{A}$ is doubly-connected, $v(c, w) \geqq 2$.

We now show, using Garabedian's upper bound and a limit argument that $v(c, w) \leqq 3$. To that end, we fix $\omega \in$ int $W_{c}$ and consider for small positive $h$ the functions $g$, analytic on $\mathfrak{A}$, taking values of modulus less than one, satisfying

$$
g(-1)=f_{c, w}(-1)=0, g(-1+h)=f_{c, w}(-1+h),
$$

and such that $g(1)$ lies on the ray issuing from $\omega$ and passing through $w$. There is a unique such $g$, which we denote by $g_{h}$, for which $|g(1)-\omega|$ is maximized. The function $g_{h}$ is an extremal relative to the point 1 for 
A. I. 596

the Pick-Nevanlinna problem specified by (3.1). By Garabedian's upper estimate the constant value of the valence of $g_{h}$ on $\Delta$ does not exceed $2+(2-1)=3$. Now

$$
f_{c, w}=\lim _{h \rightarrow 0} g_{h},
$$

and we conclude that $v(c, w) \leqq 3$.

The following theorem will play a central role in the examination of $W_{c}$.

Theorem 3.1. $\left\{v(c, w): w \in \operatorname{fr} W_{c}\right\}=\{2,3\}$ for $c$ small.

Proof. We fix $\varrho$ satisfying

$$
0<\varrho<(1-r) / 2,
$$

and note that with $\varrho$ so chosen the following three sets are mutually disjoint:

$$
\begin{aligned}
& \{|z+1| \leqq \varrho\} \\
& \{r \leqq|z| \leqq r+\varrho\}, \\
& \left\{(r+\varrho)^{-1} \leqq|z| \leqq r^{-1}\right\}
\end{aligned}
$$

There exists an analytic function $\varphi$ on $\mathfrak{U}$ satisfying

$$
-\log |\varphi|=2 G_{(-1)},
$$

$G_{a}$ denoting Green's function for $\mathfrak{A}$ with pole $a$. The assertion follows from the fact that the periods of the conjugate of $2 G_{(-1)}$ are integer multiples of $2 \pi$. We normalize $\varphi$ by the requirement that $\varphi(1)>0$.

We proceed by relating $\varphi$ and $f_{c, w}$ with small $c$. In fact, the following lemma holds:

Lemma 3.1. Given $\eta>0$, for sufficiently small $c$ there exists for each pair $(c, w), w \in \mathrm{fr} W_{c}$, a number $u, u=1$, such that

$$
\left|f_{c, u}(z)[\varphi(z)]^{-1}-u\right|<\eta, \quad z+1 \mid=\varrho .
$$

Suppose the assertion of the lemma false. Then there would exist a sequence of $f_{c, w}$ with $c$ tending to 0 , say $\left(F_{n}\right)$, which converges uniformly on compact subsets of $\mathfrak{A}$ and is such that

$$
\lim _{n \rightarrow \infty} \min _{|v|=1}\left\{\max _{|z+1|=\varrho}\left|F_{n}(z)[\varphi(z)]^{-1}-v\right|\right\}
$$

exists and is positive. We denote $\lim F_{n}$ by $F$.

Let $a \in(0,1)$. Then for each $b$ satisfying $|b| \leqq a$ the function

$$
z \rightarrow c(z+1)+b \varphi(z), z \in \mathfrak{A},
$$

belongs to $B(c)$ for $c$ small (in fact, for $c$ satisfying $a+c\left(r^{-1}+1\right) \leqq 1$ ). Setting $z=1$ in (3.4) we see that $2 c+b \varphi(1) \in W_{c}$ for $c$ so restricted. 
That is, the closed disk with center $2 c$ and radius $a \varphi(1)$ is contained in $W_{c}$. If, in addition, $2 c<a \varphi(1)$, the interior of the disk contains 0 . Hence for $c$ small, $W_{c}$ being star with respect to 0 and containing the above disk, $\left|f_{c, w}(1)\right|=|w|$ is at least as large as the distance from 0 to the point of the circumference with center $2 c$ and radius $a \varphi(1)$ which lies on the ray issuing from 0 and passing through $w$. Hence for $c$ small

$$
\left|f_{c, w}(1)\right| \geqq a \varphi(1)-2 c .
$$

We conclude with the aid of (3.5) that $|F(1)| \geqq a \varphi(1)$. Given the arbitrariness of $a$, it follows that $|F(1)| \geqq \varphi(1)$. Now $F$ is an analytic function on $\mathfrak{A}$, taking values of modulus less than one and satisfying: $F(-1)=F^{\prime}(-1)=0$. By the Lindelöf principle $F / \varphi$ takes values of modulus at most one. Since $F / \varphi$ takes a value of modulus 1 at 1 , by the maximum principle $F / \varphi$ is a constant of modulus 1 . With $u$ the value of this constant we see that $F_{n}(z) / \varphi(z)$ tends to $u$ uniformly on $\{|z+1|=\varrho\}$ and hence that (3.3) is zero. Contradiction. Lemma 3.1 is thereby established.

We now use Lemma 3.1 taking $\eta=1$ and see by Rouché's theorem that for $c$ sufficiently small $f_{c, u}$ has exactly two zeros in $\{|z+1|<\varrho\}$. They are distinct, and one of them is, of course, - 1 . We consider henceforth $c$ which are so restricted.

Now $W_{c}$ is symmetric with respect to the real axis, for if $f \in B(c)$, then also $z \rightarrow \overline{f(z)}$ belongs to $B(c)$, whence $\overline{f(1)} \in W_{c}$. Since $W_{c}$ is compact, it has a point with maximum real part. By the noted symmetry of $W_{c}$ and its convexity, there is such a point which is real; trivially, it is unique. We denote this point by $\alpha(c)$. We denote the member of $B(c)$ taking the value $\alpha(c)$ at 1 by $\psi_{c}$. Since $\psi_{c}$ has two zeros in $\{\mid z+$ $1 \mid<\varrho\}$ and $\psi_{c}(z)=\overline{\psi_{c}(z)}$, we conclude that the zero of $\psi_{c}$ which is in $\{0<|z+1|<\varrho\}$ is real.

We recall that the period of the conjugate of $G_{a}$ taken along $\{i a \mid=r\}$ negatively sensed relative to $\mathfrak{A}$ is $2 \boldsymbol{\tau}$ times the harmonic measure of $\{|z|=r\}$ with respect to $\mathfrak{A}$ at $a$ and that a corresponding result holds for $\left\{|z|=r^{-1}\right\}$. Now $-\log \mid \psi_{c}$ is a sum of Green functions and the period of its conjugate along $\{|z|=r\}$ negatively sensed relative to $\mathfrak{A}$ is $2 \pi$ times a positive integer. We conclude that $\psi_{c}$ has at least three zeros counted by multiplicity. From the fact that $v(c, w) \leqq 3$ we see that $\psi_{c}$ has exactly three zeros. They are necessarily simple and real.

For $c$ sufficiently small the zero of $f_{c, w}$ not in $\{z+1 \mid<\varrho\}$, if there is one, lies in the union of the annuli $\{r<|z|<r+\varrho\}$ and $\left\{(r+\varrho)^{-1}<|z|<r^{-1}\right\}$. This observation follows from the fact that for $c$ small the period, taken along $\{|z|=r\}$ as above, of the conjugate of $G_{a}$, $a$ being the zero of $f_{c, w}$ not in $\{|z+1|<\varrho\}$, is necessarily close to 0 
or $2 \pi$. This fact, in turn, is a consequence of (1) the zero of $f_{c, w}$ in $\{0<|z+1|<\varrho\}$ tends to -1 as $c \rightarrow 0$, and (2) the period, taken along $\{|z|=r\}$ as above, of the conjugate of $-\log \left|f_{c, w}\right|$ is $2 \pi$ times a positive integer, here necessarily 1 or 2 . The observation may also be concluded with the aid of Lemma 3.1.

We continue supposing that $c$ is further restricted to be so small that the 'third' zero of $f_{c, w}$, i.e. the one not in $\{|z+1|<\varrho\}$ lies in the union of the annuli of the preceding paragraph. We fix such an allowed $c$ and propose to study the dependence of the 'third' zero on $w \in \mathrm{fr}_{c}$. We show that for at least two values of the parameter $w$ the third zero disappears (and, subsequently, that this happens for exactly two values of $w$ ). To that end, we consider

$$
E=\left\{w \in \operatorname{fr} W_{c}: v(c, w)=3\right\} .
$$

Since $f_{c, w}$ depends continuously on $u, E$ is open in the sense of the relative topology of $\mathrm{fr}_{\boldsymbol{c}}$. We introduce $\Gamma$, the component of $E$ containing $\alpha(c)$. Now $-\alpha(c)$ is a (actually, as we shall see later, the) point of $W_{c}$ furthest left, thanks to the symmetry of $W_{c}$ with respect to 0 . It is to be observed that when $f \in B(c)$, so is $z \rightarrow-f\left(z^{-1}\right)$.

We show that $-\alpha(c) \notin \bar{\Gamma}$. To see this, we note that the 'third' zero of $f_{c, w}$ depends continuously on $w$ for $w \in \Gamma$ and hence is always in $\{r<|z|<r+\varrho\}$ or else is always in $\left\{(r+\varrho)^{-1}<|z|<r^{-1}\right\}$. Further

$$
f_{c,-\alpha(c)}(z)=-f_{c, \alpha(c)}\left(z^{-1}\right) \text {. }
$$

It follows that the 'third' zeros of $f_{c .-x(c)}$ and $f_{c, x(c)}$ are mutually reciprocal and so are not in the same one of the above annuli. From this fact and the continuous dependence of the third zero on $u$, it follows that $-\alpha(c) \notin \bar{\Gamma}$.

The proof of Theorem 3.1 is now readily completed. The set $\Gamma$ is an open subarc of fr $W_{c}$ with two distinct endpoints. The extremal of $B(c)$ corresponding to each endpoint has exactly two zeros, for otherwise the endpoint in question would belong to $\Gamma$. Recalling that $v[c, \alpha(c)]=3$, we see that Theorem 3.1 is established.

Extremals with exactly two zeros. The zero, different from -1 , of an extremal of $B(c)$ relative to 1 , having exactly two zeros, lies on $\{|z|=1\}$ as we see on considering the period of the conjugate of the Green function. We recall the well known fact that

$$
\theta \rightarrow G_{e^{i \theta}}(-1)
$$

is increasing on $\{0 \leqq \theta \leqq \pi\}$. Using this fact and the relation

$$
G_{a}(-1)=G_{\bar{a}}(-1), a \in \mathfrak{A},
$$


we see that there are at most two extremals of $B(c)$ relative to 1 which have exactly two zeros. Since the extremals corresponding to the endpoints of $\Gamma$ have this property, we see that there are exactly two such extremals. The extremals corresponding to the points of fr $W_{c}-\bar{\Gamma}$ have three zeros.

Now the set of extremals of $B(c)$ relative to 1 is mapped univalently onto itself by both

$$
f \rightarrow\left(z \rightarrow-f\left(z^{-1}\right)\right)
$$

and

$$
f \rightarrow(z \rightarrow \overline{f(\bar{z})})
$$

The maps (3.6) and (3.7) preserve the constant value of the valence of an extremal. Hence the set of values taken at 1 by the extremals of valence 2 is mapped onto itself by $z \rightarrow-z$ and $z \rightarrow \bar{z}$. Since neither of these values is real, we see that the set is of the form

$$
\{i \beta(c),-i \beta(c)\}, 0<\beta(c)<1 .
$$

It may now be concluded that

$$
\Gamma=\{\operatorname{Re} z>0\} \cap \text { fr } W_{c}
$$

and that $\Gamma$ is mapped onto itself by $z \rightarrow \bar{z}$ and onto fr $\Pi_{c}-\bar{\Gamma}$ by $z \rightarrow-z$.

\section{4. $\mathrm{W}_{c}$ is not a circular disk for $\mathrm{e}$ small}

We continue with $c$ subject to the stated restrictions. If $W_{c}$ were a circular disk, its center would be zero since $W_{c}$ is symmetric with respect to 0 . This would imply that $\alpha(c)=\beta(c)$. But we shall show that for $c$ sufficiently small

$$
\alpha(c)<\beta(c) .
$$

Thus we shall conclude

Theorem 4.1. $W_{c}$ is not a circular disk for $c$ small.

Proof of (4.1). Let $u$ denote the extremal in $B(c)$ relative to 1 which has valence of constant value 2 on 1 whose zero $\eta, \neq-1$, has positive imaginary part. Let $v$ denote an extremal in $B(c)$ relative to 1 which takes a real value at 1 . Let $a$ denote the zero of $v$ lying in $\{0<|z+1|<\varrho\}$ and let $b$ denote the other zero of $v$ which is different from - 1. We suppose, as we may with the aid of (3.6), that $v$ is so normalized that $r<|b|<r+\varrho$. 
We note the following relations:

$$
\begin{gathered}
-\log |u|=G_{\eta}+G_{(-1)}, \\
-\log |v|=G_{a}+G_{b}+G_{(-1)} .
\end{gathered}
$$

The parameters $\eta, a, b$ are subject to certain restrictions as we shall now see. We introduce

$$
A=\lim _{z \rightarrow-1}\left[G_{(-1)}(z)+\log |z+1|\right] .
$$

From (4.2) and (4.3) we obtain, using the condition on the derivative at -1 for members of $B(c)$,

$$
G_{\eta}(-1)+A=G_{a}(-1)+G_{b}(-1)+A=-\log c .
$$

From the fact that the constant value of the valence of $v$ on $\Delta$ is 3 , we conclude on calculating the period along $\{|z|=r\}$ of the conjugate of $-\log |v|$ that

$$
\frac{\log (a \mid r)}{\log r^{2}}+\frac{\log (b r)}{\log r^{2}}
$$

is either $1 / 2$ or $3 / 2$. By the normalization of $v$ the second term in the sum exceeds $1 / 2$. Hence the sum has the value $3 / 2$. We conclude

$$
|a b|=r .
$$

The proof of (4.1) will now be completed with the use of the behavior of $G_{1}$ near -1 and of the symmetry of the Green function. Indeed, the number of zeros in $\mathfrak{U}$ counted by multiplicity of $\left(G_{1}\right)_{z}$ is 1 since $\mathfrak{A}$ is a doubly-connected region with non-degenerate boundary components. Since $G_{1}\left(z^{-1}\right)=G_{1}(z), z \in \mathfrak{U},\left(G_{1}\right)_{z}$ has its zero at -1 . We conclude that

$$
G_{1}(z)=G_{1}(-1)+B\left[(\operatorname{Re} z+1)^{2}-(\operatorname{Im} z)^{2}\right]+o\left(|z+1|^{2}\right), \quad B \neq 0 .
$$

Now $B<0$ since the restriction of $G_{1}$ to the segment $\left[-r^{-1},-r\right]$ attains its maximum at the zero of $\left(G_{1}\right)_{z}$, i.e. at -1 . U sing (4.2), (4.3), the symmetry of the Green function, and (4.6) we obtain

$$
\begin{aligned}
\log \frac{\beta(c)}{\alpha(c)} & =G_{1}(a)+G_{1}(b)-G_{1}(\eta) \\
& >B(a+1)^{2}+o\left[(a+1)^{2}\right]+G_{1}(b) \\
& +B(\operatorname{Im} \eta)^{2}+o\left[\eta \eta+\left.1\right|^{2}\right] .
\end{aligned}
$$

From the boundary behavior of $G_{1}$ and the minimum principle for harmonic functions, with 


$$
C=\left[\min _{|z|=r+\varrho} G_{1}(z)\right] / \log \left(1+\varrho r^{-1}\right)
$$

we have

$$
G_{1}(z) \geqq C \log \frac{|z|}{r}, r<|z|<r+\varrho .
$$

Using (4.5) we conclude

$$
G_{1}(b) \geqq C \log \frac{1}{|a|} .
$$

Further, using (4.4) and the symmetry of the Green function, we obtain

$$
\log |\eta+1|+o(1)=\log (a+1)+o(1)-G_{(-1)}(b) .
$$

We conclude from (4.9) and (4.5) that

$$
\lim _{c \rightarrow 0}\left|\frac{\eta+1}{a+1}\right|=1 \text {. }
$$

Hence from (4.7) and (4.8) we obtain

$$
\log \frac{\beta(c)}{\alpha(c)}>C \log \frac{1}{|a|}+3 B(a+1)^{2}>0
$$

for $c$ sufficiently small.

The inequality (4.1) follows, and Theorem 4.1 is thereby established.

\section{The case of zero order interpolation}

In this section we show by continuity considerations that the Grenzkreis phenomenon does not persist in the case of zero order interpolation. To that end, we introduce an auxiliary function, $\theta(z)=1-z^{2}, z \in \mathfrak{A}$. We fix $c$ positive and so small that

$$
c \sup |\theta|<1
$$

and (4.1) holds. We consider the Pick-Nevanlinna interpolation problem for $\mathfrak{A}$ with the interpolation requirements

$$
f(-1)=0, f(-1+h)=c h,
$$

where $0<h<1-r$. Let $V_{h}$ denote the set of values taken at 1 by the functions $f$, analytic on $\mathfrak{A}$, taking values of modulus less than one, and satisfying (5.2). It is our object to show that for $h$ sufficiently small $V_{h}$ is a set with nonempty interior but is not a circular disk.

We introduce $m$ satisfying 


$$
\alpha(c) / \beta(c)<m<1
$$

and consider the functions

$$
m \varphi+\left[\frac{c h-m \varphi(-1+h)}{\theta(-1+h)}\right]^{\theta}, \varphi \in B(c),
$$

which satisfy the interpolation conditions (5.2). There exists $h_{0}$, $0<h_{0}<1-r$, such that

$$
\left|\frac{c h-m \varphi(-1+h)}{\theta(-1+h)}\right|<c(1-m),
$$

when $0<h \leqq h_{0}$. For such $h$ the functions (5.3) take values of modulus less than one and we proceed understanding that $h$ is so restricted. Since $\theta(1)=0, m \alpha(c),-m \alpha(c), m \beta(c) i,-m \beta(c) i \in V_{h}$. Further $0 \in \operatorname{int} V_{h}$.

Let $\mu(h)$ denote the maximum real point of $V_{h}$ and let $\lambda(h)$ denote the minimum real point of $V_{h}$. We first show that $\lim _{h \rightarrow 0} \mu(h)=\alpha(c)$ and $\lim _{h \rightarrow 0} \lambda(h)=-\alpha(c)$. In fact, given $t, 0<t<1$, we have $\mu(h)>t \alpha(c)$, $\lambda(h)<-t \alpha(c)$ for $h$ small (as we see on introducing an allowed $m>t$ ). Further there exists $\varphi \in B(c)$ such that $\phi(1)$ is real and at least as large as $\lim \sup \mu(h)$. We conclude that $\lim \mu(h)=\alpha(c)$. Similarly, we conclude that $\lim \lambda(h)=-\alpha(c)$.

If $V_{h}$ were a circular disk, the center of $V_{h}$ would be real, the points of $\operatorname{fr} V_{h}$ on the imaginary axis being the negative of one another since $V_{h}$ is mapped onto itself under $z \rightarrow \bar{z}$. The radius would be $\left[\mu(h)-\lambda_{h}(h)\right] 2$ and so

$$
\left[\mu(h)-\lambda_{(}(h)\right] / 2 \geqq m \beta(c) .
$$

Hence if $V_{h}$ were a circular disk for arbitrarily small $h$, we would have

$$
\alpha(c) \geqq m \beta(c) \text {. }
$$

From this contradiction we conclude that $V_{h}$ is not a circular disk for small positive $h$. The Grenzkreis phenomenon is seen not to persist for zero order interpolation.

\section{Positive results concerning the Wertevorrat in the case of an annulus}

A Pick-Nevanlinna interpolation problem for a Riemann surface may be referred with the aid of a conformal universal covering to the case of the unit disk and be reduced to a problem concerning interpolation by analytic functions on $\Delta$ which take values of modulus at most one and are automorphic with respect to the Fuchsian group of conformal auto- 
morphisms of $\Delta$ leaving the conformal universal covering invariant. [For this reduction, cf. [4] where only the case of an annulus and of zero order interpolation is considered. The discussion is, however, applicable to the general situation and prepares the way for the developments of the following paragraph.] With the aid of this reduction, in the situation where the problem on the Riemann surface has more than one solution, the question of the Wertevorrat may be reformulated as follows thanks to uniqueness considerations given by $\mathrm{R}$. Nevanlinna in his memoir on Pick-Nevanlinna interpolation [11].

Given a Riemann surface $S$, a Pick-Nevanlinna interpolation problem on $S$ having more than one solution, and $\varphi$, a conformal universal covering of $S$ having domain $A$, there exist functions $A, B, C$, analytic on $\Delta$, taking values of modulus less than one, such that

$$
w \rightarrow \mathbf{A}_{z}(w)=\frac{A(z) w+B(z)}{C(z) w+1}
$$

maps $\bar{A}$ into $A$ for each $z \in A$ and $A-B C$ is not the constant 0 , having the property that the interpolation functions for the given PickNevanlinna problem on $S$ composed with $\varphi$ are given exactly by the functions,

$$
z \rightarrow \mathbf{A}_{z}[g(z)], \quad|z|<1,
$$

where the $g$ are those functions analytic on 1 , taking values of modulus at most one and satisfying the srstem of equations

$$
g \circ \tau=[\operatorname{inv} \alpha(\tau)] \circ g,
$$

for all $\tau \in \Gamma$, the group of conformal automorphisms of $\Delta$, leaving $\varphi$ invariant, $\alpha(\tau)$ being the Möbius transformation*) mapping $\bar{\Delta}$ onto itself and satisfying

$$
\mathbf{A}_{\tau(z)}(w)=\mathbf{A}_{z}[\alpha(\tau)(w)]
$$

$|z|<1,|w| \leqq 1$. Thus, the Wertevorrat question is referred to the question of studying the image with respect to (6.1) of the set of $g(z)$ at a non interpolation point $z, g$ being an alloued solution of (6.3). In the case where $\Gamma$ is not abelian, the precise nature of the Wertevorrat appears to be elusive. However, the situation for a non-degenerate annulus is particularly favorable, for here $\Gamma$ is generated by a hyperbolic conformal automorphism of $\Delta$.

*) We need to allow the possibility of constant $g$ taking a value having modulus one. Hence the distinction made relative to $\alpha(\tau)$. 
We shall see that in the case of a non-degenerate annulus either for all non-interpolation points $z$ the Wertevorrat is a closed circular disk $D(z) \subset \Delta$, or for all non-interpolation points $z$ the Wertevorrat is the intersection $I(z)$ of two closed circular disks, each contained properly in $\bar{\Delta}$, their circumferences having exactly two points in common.

The problem may be referred to the nature of $\alpha(\tau)$ where $\tau$ is a generator of $\Gamma$. Since $\alpha(\tau)$ maps $\bar{\Delta}$ onto itself, $\alpha(\tau)$ is non-loxodromic. Simple interpolation situations yield examples where $\alpha(\tau)$ is the identity or elliptic (e.g. the problem on $\mathfrak{A}$ with (1) $f(-1)=f^{\prime}(-1)=0$, resp. (2) $f(-1)=0)$. The examples of $\S \S 4,5$ will show that a hyperbolic $\alpha(\tau)$ is realizable. We have not decided whether parabolic $\alpha(\tau)$ are realizable. The proof of the assertion of the previous paragraph, to which we now turn, does not depend on the answer to the question.

If $\alpha(\tau)$ is the identity, the set of $g(z)$ is $\bar{\jmath}$. If $\alpha(\tau)$ is elliptic, the problem may be reduced to the case where the fixed points of $\alpha(\tau)$ are 0 and $\infty$. A direct examination then shows that when $\alpha(\tau)$ is elliptic, the set of $g(z)$ is a closed circular disk of positive radius lying in $\Delta$. If $\alpha(\tau)$ is parabolic, the problem may be reduced with the aid of a suitable Möbius transformation mapping $\Delta$ onto $\{\operatorname{Re} z>0\}$ to the study of the set of values at $z \in \Delta$ of the solutions $h$ of

$$
h \circ \tau=h+i,
$$

$h$ being either the constant $\infty$ or else analytic on $\Delta$ and taking values with positive real part. The set of $h(z)$ is of the form

$$
\{\operatorname{Re} z \geqq c(>0)\} \cup\{\infty\}
$$

and consequently the set of $g(z)$ is a closed circular disk contained properly in $\bar{J}$, the circumference of which is tangent to the unit circumference at the fixed point of $\alpha \cdot(\tau)$.

We now conclude that in the three cases considered thus far the Wertevorrat at a non-interpolation point is a closed circular disk of positive radius in $\Delta$.

It now follows, thanks to the example of $\S 4$, that the case of hyperbolic $\alpha(\tau)$ is indeed realized, the Wertevorrat not being a circular disk.

The case where $\alpha(\tau)$ is hyperbolic may be reduced with the aid of suitable Möbius transformations to the study of

$$
h(\lambda z)=\mu h(z),
$$

where $1<\lambda, \mu<+\infty$, and solutions $h$ are considered having domain $\{\operatorname{Re} z>0\}$ which are the constant 0 , the constant $\infty$, or else are analytic maps of $\{\operatorname{Re} z>0\}$ into itself. One concludes with the aid of the lemma of 
Julia-Wolff-Carathéodory that a necessary condition for (6.6) to have allowed solutions other than the constants 0 and $\infty$ is that

$$
\mu \leqq \lambda
$$

That it is sufficient may be seen with the aid of

$$
\exp \left(\frac{\log \mu}{\log \lambda} \log z\right),
$$

where $» \log »$ denotes the principal logarithm. Further, in case $\mu=\lambda$, the only non-trivial solutions are of the form $c z, c$ a positive number, as we see with the aid of the lemma of Julia-Wolff-Carathéodory. Cf. [5]. Given $z_{0}$ satisfying $\operatorname{Re} z_{0}>0$, the set of $h\left(z_{0}\right)$ for given $\lambda$ and $\mu, \mu \leqq \lambda$, is of the form

$$
\{\beta \leqq \operatorname{Arg} z \leqq \gamma\} \cup\{0, \infty\},
$$

where $-\pi / 2<\beta \leqq \gamma<\pi / 2$, as we see by considering the maximum and minimum of $\operatorname{Arg} h\left(z_{0}\right)$ for solutions $h$ satisfying $\left|h\left(z_{0}\right)\right|=1$ and using the fact that the family of allowed $h$ is positive homogeneous and closed with respect to addition. Here »Arg» denotes the principal argument. We remark that $\beta=\gamma$ exactly when $\mu=\lambda$. Cf. (6.8) and the observation following (6.8).

Returning to the situation for the unit disk, we see that for the uniformized version of the interpolation problem only multipliers $\mu<\lambda$ arise. This is so, because when there is more than one interpolating function, the Wertevorrat at a non-interpolating point, having the Study property, has a non-empty interior. Cf. $\S 1$ of this paper, [13], [8], and [9]. One may also use the results of $\S 8$ of this paper.

We are led to the conclusion that when $\alpha(\tau)$ is hyperbolic, the Wertevorrat at a non-interpolation point is a closed convex lune lying in $\Delta$, the frontier of which is the union of two circular arcs, or a circular arc and a rectilinear segment, having common endpoints. The second alternative is excluded by the Study property. Cf. [8], [13]. It is a direct consequence of the theorem of p. 172 and the lemma of p. 173 of [8] that the arcs lie in closed disks bounded by oricycles to which they are tangent. We are referring to the representation of Minkowskian type given in [8] for proper subsets of $\Delta$, which are closed in the sense of the relative topology of $\Delta$ and have the Study property. We see that the circumferences containing the circular arcs lie in the closed disks in question. The Wertevorrat is the intersection of the closed disks bounded by these circumferences. The asserted property of the Wertevorrat is seen to follow. 


\section{Precisions on $\mathbf{W}_{c}$ of $\S 4$, $\mathrm{c}$ small}

We now see that $W_{c}$ is bounded by the union of two circular arcs having common endpoints. More precisely, they are the circular arcs with endpoints $i \beta(c),-i \beta(c)$ which contain respectively $\alpha(c)$ and $-\alpha(c)$. This observation is a consequence of the fact that $z \rightarrow \bar{z}$ and $z \rightarrow-z$ map $W_{c}$ onto itself, which, taken with the fact that the endpoints are characterized by being corners, implies that it is not the case that one endpoint has a non-zero real part and the other has a non-zero imaginary part (for otherwise, there would be four distinct points in the set of endpoints). There remains to exclude the case where the endpoints are $\alpha(c)$ and $-\alpha(c)$. It cannot occur since the disk bounded by the circumference passing through $\alpha(c),-\alpha(c)$, $i \beta(c)$ does not contain - $i \beta(c)$. The asserted property of $W_{c}$ follows.

\section{Some general facts concerning Pick-Nevanlinna interpolation}

In this section we give an account of the results we have used concerning Pick-Nevanlinna interpolation with a finite set of data in a setting of Riemann surface theory. We are proceeding under the assumption that the given Riemann surface has finite topological characteristics, is hyperbolic, and that the boundary components are non-degenerate. Consequently, we suppose, as we may, that the given surface is a region $\Omega$ of a compact Riemann surface $S$, the frontier $\Gamma$ of $\Omega$ consisting of a finite number of mutually disjoint closed Jordan curves which are regular and analytic, that $\Gamma=\operatorname{fr}(S-\bar{\Omega})$, and that there exists a univalent anticonformal map $\alpha$ of $S$ onto itself keeping the points of $T$ fixed and mapping $\Omega$ onto $S-\bar{\Omega}$. Thus $S$ is a Schottky double of $\Omega$. Our objectives are to show that when there is more than one interpolating function, (1) the Wertevorrat at a noninterpolation point $b$ has a nonempty interior, and (2) there is a unique interpolating function taking at $b$ an assigned value lying in the frontier of the Wertevorrat and it has the constant valence property. We also show (3) the Garabedian bound extends in a natural way.

We shall call a Pick-Nevanlinna problem determinate provided that it has exactly one solution. In the case of the problem we are considering on $\Omega$ (with a finite set of data) determinacy implies, as a consequence of the exchange principle given on pp. 63,64 of [7] that the solution is a unitary function (Einheitsfunktion) in the sense of Carathéodory [2], i.e. is the restriction to $\Omega$ of a meromorphic function on $S$ which is analytic in $\Omega$ and takes values of modulus one on $\Gamma$. It is classical that a non-constant 
analytic function on $\Omega$ taking values of modulus less than one has constant finite valence on $\Delta$ if and only if it is unitary.

We continue under the assumption that the set Pick-Nevanlinna problem has more than one solution. Let $W$ denote the Wertevorrat of the family of solutions at the non-interpolation point $b$. Let $w \in W$. We consider the augmented problem requiring that, in addition to the conditions already put down, a solution $f$ satisfy: $f(b)=w$. The following theorem holds.

Theorem 8.1. If the augmented problem is not determinate, then $w \in$ int $W$.

The stated properties of the Wertevorrat will be seen to follow.

The proof to be given of Theorem 8.1 will be based on the exchange principle and is related to the one we used for the zero order case. Cf. [6, p. 572]. However, the auxiliary polynomial introduced there is not adequate for interpolation of higher order. To construct for the present purposes a convenient auxiliary function, we fix $q \in S-\bar{\Omega}, q$ being a non-Weierstrass point of $S$, and note the existence of functions analytic on $S-\{q\}$ having an assigned simple zero but no other zeros. The existence of such 'primary' functions is assured by construction with the aid of harmonic functions having singularities of an elementary kind at $q$, resp. at $q$ and the assigned point. With the aid of the reciprocals of such primary functions, we may construct a meromorphic function $\varphi$ on $S-\{q\}$ having at a finite number of assigned points of $S-\{q\}$ assigned principal parts (possibly 0) relative to fixed local uniformizers for these points. Suppose now that $A$ is a finite subset of $S-\{q\}$ and $B$ is a finite subset of $S-(\{q\} \cup A)$. We introduce a function $\psi$, the product of a finite number of primary functions, having zeros of assigned positive orders at the points of $A$, and zeros at the points of $B$. We now introduce $\varphi$ as above, which is analytic at the points of $S-(\{q\} \cup A)$ and has principal parts at the points of $A$ in ter ms of the fixed local uniformizers which are such that the initial Taylor section of $\psi \psi$ (taken in terms of the uniformizer) of order equal to the multiplicity of $\psi$ at the point less one has assigned coefficients.

We now turn to the proof of Theorem 8.1. Using the exchange principle we see that there are two distinct unitary functions, sar $f_{1}$ and $f_{2}$. satisfying the conditions of the augmented problem. Let $f=\left(f_{1}+f_{2}\right)_{2}$. We let $A$ denote the union of $\{b\}$ and the set of points at which the set PickNevanlinna problem specifies interpolation conditions, and we let $B$ denote the finite set on $\Gamma$ at the points of which $f$ takes ralues of modulus one. We specialize $\psi$ to have zeros at the points of $A$ having orders equal to the order of the interpolation condition of the augmented problem plus one and zeros at $B$. We thereupon choose $\varphi$ so that $\varphi \psi$ satisfies the interpolation conditions of the augmented problem.

For $t$ sufficiently small and positive 


$$
g=(1-t) f+t \varphi \psi
$$

satisfies the interpolation conditions of the augmented problem and also

$$
\max _{\bar{\Omega}}|g|<1 .
$$

We fix such $t$. Now let $\theta$ be a product of primary functions having zeros at the points of interpolation of the originally given Pick-Nevanlinna problem, the order being the order of interpolation plus one, but no other zeros. On considering

$$
g+t \theta
$$

for small complex $t$, we conclude that $w \in$ int $W$. Theorem 8.1 is thereby established.

It is now easy to conclude the stated properties (1) and (2) of the Wertevorrat.

(1) Suppose that int $W=\phi$. Let $f_{1}, f_{2}$ satisfy the set Pick-Nevanlinna problem and let $f_{3}=\left(f_{1}+f_{2}\right) / 2$. The augmented problem with the supplementary condition $f(b)=f_{k}(b)$ where $k$ takes one of the values $1,2,3$ is determinate since int $W=\phi$. The three functions $f_{1}, f_{2}, f_{3}$ must be unitary functions. Consequently, $f_{3}$ taking boundary values of modulus one on $\Gamma, f_{1}=f_{2}$. It follows that there is only one solution to the set Pick-Nevanlinna problem. Contradiction. We conclude that int $W \neq \phi$.

(2) This is immediate by Theorem 8.1, since, when $w \in$ fr $W$, trivially $w \notin$ int $W$. By the observations made above the solution is unitary.

(3) The Garbedian bound. We consider a determinate Pick-Nevanlinna problem relative to $\Omega$ with a finite set of data and denote the solution by $f$. We seek to obtain a Garabedian upper bound for the number of zeros of $f$, which is the constant value of the valence of $f$ on the open unit disk when $f$ is not constant. (We put aside the trivial case where $f$ is constant.) Now $f$ is the unique analytic function on $\Omega$ satisfying the stated interpolation conditions and having the least sup norm of those which do. Indeed, the minimum of the sup norms of the functions in question cannot be less than one, for otherwise the stated Pick-Nevanlinna problem would not be determinate. For an interpolation point $b$ we let $v(b)$ denote the order of interpolation at $b$ augmented by one. We let $v$ denote the sum of the $v(b)$ taken over the interpolation points $b$. The Euler characteristic of $\Omega$ will be denoted by $\chi$. We shall show

Theorem 8.2. $f$ has at most $v+\%$ zeros counted by multiplicity.

We note that, thanks to (2), Theorem 8.2 subsumes the upper estimate of Garabedian, for in the situation which he studied $\chi=n-2$ and $v=k+1$. Cf. the result quoted in the third paragraph of $\S 1$ of this paper. The lower estimate of the valence in the non-constant case as the number 
of boundary contours does not call for special comment. [In the situation where some boundary components may be pointlike but not all, it suffices to consider the surface obtained by the adjunction of points corresponding to the pointlike boundary components and to observe that determinacy relative to the stated problem holds or fails simultaneously for the surface and its extension. The upper bound for the valence of a determinate relative to the extended surface yields for the valence of a determinate relative to the given surface an ameliorated upper bound which takes into account the number of pointlike boundary components. One must, of course, reckon on the valence being smaller at the points of $\Delta$ corresponding to the pointlike boundary components. Correspondingly, one also obtains a lower bound subject to suitable qualification.]

Proof of Theorem 8.2. We shall reduce the question to that of obtaining an upper bound on the number of zeros, counted according to multiplicity, of $H_{p}$ minimizers satisfying the stated interpolation requirements, $1 \leqq p<+\infty$. The latter question will be treated with the aid of a variational formula of F. Riesz [15] and the theorem of Cauchy-Read [14]. For the basic facts concerning Hardy classes on Riemann surfaces, cf. [7]. Given $F$ analytic on $\Omega, F$ is said to belong to the Hardy class $H_{p}(\Omega)$ provided that $\mid F^{p}$, which is subharmonic, has a harmonic majorant (resp. to $H_{s_{0}}(\Omega)$ when $F$ is bounded). We fix $a \in \Omega$ and define the $H_{p}$ norm of $F \in H_{p}(\Omega)$ as the $p$ th root of the value at $a$ of the least harmonic majorant of $|F|^{p}$. It is a standard fact that the $H_{p}$ norm of $F$ is given by

$$
\left(\frac{i}{2 \pi} \int_{\Gamma}\left|F^{*}\right| p \delta g_{a}\right)^{1 / p}
$$

where $F^{*}$ is the Fatou boundary function of $F, g_{a}$ is Green's function for $\Omega$ with pole $a$, and $\delta g_{a}$ is the abelian differential given in terms of a local uniformizer $\theta$ by $2\left(g_{a} \circ \theta\right)_{z} d z$. Cf. [7]. We consider for a given $p$ the member(s) of $H_{p}(\Omega)$ satisfying the interpolation conditions of the set Pick-Nevanlinna problem with which we are concerned and having minimum norm. [When $1<p<+\infty$, there is uniqueness. When $p=1$, the question of uniqueness is not settled, as far as I am aware. However, we shall have no need to concern ourselves with uniqueness questions.] These minimizers tend to $f$, the unique solution of the determinate problem, when $p \rightarrow \infty$, as we see with the aid of the qualitative Harnack inequality for $\Omega$. We thus see that the proof of Theorem 8.2 reduces to showing that for a given $p$ the number of zeros, counted by multiplicity, of a member of $H_{p}(\Omega)$ satisfying the stated interpolation conditions which has minimum $H_{p}$ norm does not exceed $v+\chi$.

Let $I$ denote the vinterpolation» divisor defined on $\Omega$ by $I(b)=v(b)$ 
A. I. 596

at points of interpolation $b$ and by $I(c)=0$ at the remaining points $c$ of $\Omega$. The cited variational formula of F. Riesz, which was given by him for the case $p=1$ and $\Omega=\Delta$ but is available suitably modified when $1 \leqq p<+\infty$ and $\Omega$ is not specialized, yields the result that for all $\varphi \in H_{p}(\Omega)$ such that $\partial_{\varphi}$, the divisor of $\varphi$, satisfies

$$
\partial_{\varphi} \geqq I \text {, }
$$

we have

$$
\int_{\Gamma}\left|F^{*}\right|^{p-1} s g\left(\overline{F^{*}}\right) \varphi^{*} \delta g_{a}=0 .
$$

Let $\partial$ denote $-\left(\partial_{\delta g_{a}}+I\right)$, where $\partial_{\delta g_{a}}$ is the divisor of $\delta g_{a}$ (on $\Omega$ ). Let $q$ denote $p /(p-1)$ when $1<p<+\infty$, and $+\infty$ when $p=1$. Let $H_{q, \partial}(\Omega)$ denote the class of meromorphic functions $\psi$ on $\Omega$ satisfying: (1) $\partial_{\psi} \geqq \partial$, and (2) the function

$$
|\psi| \exp \left[\sum_{c \in \Omega} \partial(c) g_{c}\right]
$$

is bounded, $g_{c}$ being Green's function for $\Omega$ with pole $c$, when $q=+\infty$, and its $q$ th power, which is subharmonic, has a harmonic majorant, when $1<p<+\infty$. [For the class $H_{q, \partial}(\Omega)$, ef. [7, p. 79].] It follows from the theorem of Cauchy-Read that $\left|F^{*}\right|^{p-1} s g\left(\overline{F^{*}}\right)$ is the Fatou boundary function of a function $G \in H_{q, \partial}(\Omega)$.

Now $F G \in H_{1, \partial}(\Omega)$ and further has real (non-negative) Fatou boundary values. Hence $F G$ is the restriction of a function $M$ meromorphic on $S$ which takes finite non-negative real values on $I$. Further $M$ is not the constant zero since not all interpolated values are zero. Since $G \in H_{q, \partial}(\Omega)$, we conclude that $F$ and $G$ each have only a finite number of zeros. On appeal to the reflexion property of $\lambda$ under the anti-conformal map $\alpha$ introduced in the first paragraph of this section, namely

$$
M \circ \alpha=\bar{I},
$$

we see that

$$
\sum_{c \in \Omega}\left[\partial_{F}(c)+\partial_{G}(c)\right] \leqq 0 .
$$

Thus we see that the number of zeros of $F$ in $\Omega$, counted by multiplicity, does not exceed $-\sum_{c \in \Omega} \partial_{G}(c)$. Since $G \in H_{q, \Omega}(\Omega)$, we have

$$
\sum_{c \in \Omega} \partial_{G}(c) \geqq \sum_{c \in \Omega} \partial(c)=-(v+\%) .
$$

Thus the number of zeros of $F$, counted by multiplicity, does not exceed $v+\chi$ as we wished to show. Theorem 8.2 follows.

University of Maryland

Department of Mathematics

College Park, Maryland 20742, USA 


\section{References}

[1] Ahlfors, L.: Open Riemann surfaces and extremal problems on compact Riem£nn surfaces. - Comment. Math. Helv. 24 (1950), 100-133.

[2] Carathéodory, C.: Funktionentheorie II. - Birkhäuser Verlag, Basel, 1950.

[3] Garabedian, P. R.: Schwarz's lemme and the Szegö kernel function. - Trans. Amer. Math. Soc., $6 \%$ (1949), 1-35.

[4] Heins, M.: Extremal problems for functions analytic and single-valued in a doubly-connected region. - Amer. J. Math. 62 (1940), 91-106.

[5] - - A generalization of the Aumann-Carathéodory "Starrheitssatz». - Duke Math. J. 8 (1941), 312-316.

[6] - - A lemma on positive harmonic functions. - Ann. of Math. 52 (1950), 568 573.

[7] - -- Hardy classes on Riemann surfaces. - Lecture Notes in Math. 98, Springer Verlag, Berlin - Heidelberg - New York, 1969.

[8] - $\gg-$ On a theorem of Study concerning conformal maps with convex images. Mathematical Essays Dedicated to A. J. Macintyre. Edited by Hari Shankar. - Ohio University Press, Athens, Ohio, 1970.

[9] - - - On a theorem of Study concerning conformal maps with convex images II. - Math. Scand. 32 (2) (1973).

[10] Nevanlinsa, R.: Ueber beschränkte analytische Funktionen, die in gegebenen Punkten vorgeschriebene Werte annehmen. - Ann. Acad. Sci. Fenn. B XV (1919), $71 \mathrm{pp}$.

[11] -»- Ueber beschränkte analytische Funktionen. - Ibid. A XXXII (1929), $75 \mathrm{pp}$.

[12] Pick, G.: Ueber die Beschränkungen analytischer Funktionen, welche durch vorgegebene Funktionswerte bewirkt werden. - Math. Ann. 77 (1916), $7-23$.

[13] Pommerenke, C.: Images of convex domains under convex conformal mappings. Michigan Math. J. 9 (1962), p. 257.

[14] READ, A.: A converse of Cauchy's theorem and applications to extremal problems. - Acta Math. 100 (1958), 1-22.

[15] Riesz, F.: Ueber Potenzreihen mit rorgeschriebenen Anfangsgliedern. - Acta Math. 42 (1920), 145-171.

[16] WALSH, J. L.: Interpolation and approximation by rational functions in the complex domain. - Colloq. Publications of the Amer. Math. Soc., 20, Providence, 3d. ed., 1960. 